ADP-15-28/T930

DESY 15-153

Edinburgh 2015/18

Liverpool LTH 1053

August 2015

\title{
Isospin splittings of meson and baryon masses from three-flavor lattice QCD + QED
}

\author{
R. Horsley ${ }^{a}$, Y. Nakamura ${ }^{b}$, H. Perlt ${ }^{c}$, D. Pleiter ${ }^{d}$, P. E. L. Rakow ${ }^{e}$, \\ G. Schierholz ${ }^{f}$, A. Schiller ${ }^{c}$, R. Stokes ${ }^{g}$, H. Stüben ${ }^{h}$, \\ R. D. Young ${ }^{g}$ and J. M. Zanotti ${ }^{g}$ \\ ${ }^{a}$ School of Physics and Astronomy, University of Edinburgh, \\ Edinburgh EH9 3FD, United Kingdom \\ ${ }^{b}$ RIKEN Advanced Institute for Computational Science, \\ Kobe, Hyogo 650-0047, Japan \\ ${ }^{c}$ Institut für Theoretische Physik, Universität Leipzig, \\ 04103 Leipzig, Germany \\ ${ }^{d}$ Jülich Supercomputer Center, Forschungszentrum Jülich, \\ 52425 Jülich, Germany \\ ${ }^{e}$ Theoretical Physics Division, Department of Mathematical Sciences, \\ University of Liverpool, Liverpool L69 3BX, United Kingdom \\ ${ }^{f}$ Deutsches Elektronen-Synchrotron DESY, \\ 22603 Hamburg, Germany \\ ${ }^{g}$ CSSM, Department of Physics, University of Adelaide, \\ Adelaide SA 5005, Australia \\ ${ }^{h}$ RRZ, University of Hamburg, 20146 Hamburg, Germany
}

\begin{abstract}
Lattice QCD simulations are now reaching a precision where isospin breaking effects become important. Previously, we have developed a program to systematically investigate the pattern of flavor symmetry beaking within QCD and successfully applied it to meson and baryon masses involving up, down and strange quarks. In this Letter we extend the calculations to QCD + QED and present our first results on isospin splittings in the pseudoscalar meson and baryon octets. In particular, we obtain $M_{\pi^{+}}-M_{\pi^{0}}=4.60(20) \mathrm{MeV}$ and $M_{n}-M_{p}=1.35(18) \mathrm{MeV}$.
\end{abstract}




\section{Introduction and general strategy}

Isospin breaking effects are crucial for the existence of our Universe. Our Universe would not exist in the present form if the $n-p$ mass difference would only be slightly different. If it would be larger than the binding energy of the deuteron, no fusion would take place. If it would be a little smaller, all hydrogen would have been burned. Isospin breaking in hadron masses has two sources, the mass difference of up and down quarks, and electromagnetic interactions. Both effects are of the same order of magnitude and cannot be separated unambiguously due to the nonperturbative nature of the strong interactions. This makes a direct calculation from QCD + QED necessary [1].

In [2, 3] we have outlined a program to systematically investigate the pattern of flavor symmetry breaking in three-flavor lattice QCD for Wilson-type fermions. Our strategy was to start from the SU(3) symmetric point with all three quark masses equal, $m_{u}=m_{d}=m_{s}$, and extrapolate towards the physical point keeping the average sea quark mass $\bar{m}=\left(m_{u}+m_{d}+m_{s}\right) / 3$ constant. For this trajectory to reach the physical quark masses, $\bar{m}$ is tuned to the physical value of the average pseudoscalar meson mass $X_{\pi}^{2}=\left(M_{K^{0}}^{2}+M_{K^{+}}^{2}+2 M_{\pi^{0}}^{2}-M_{\pi^{+}}^{2}\right) / 3$. We denote the distance from $\bar{m}$ by

$$
\delta m_{q}=m_{q}-\bar{m}, \quad q=u, d, s .
$$

This implies $\delta m_{u}+\delta m_{d}+\delta m_{s}=0$ on our quark mass trajectory. To describe how physical quantities depend on the quark masses, we Taylor expand about the symmetric point [3]. This results in polynomials in $\bar{m}$ and $\delta m_{q}$, which we classify into representations of the $\mathrm{SU}(3)$ and $\mathrm{S}_{3}$ flavor groups. As we keep $\bar{m}$ constant and change only the octet part of the mass matrix, to first order in $\delta m_{q}$ flavor symmetry is broken by an SU(3) octet, leading to Gell-Mann-Okubo mass relations. We follow a similar approach here with QED added [4].

The symmetry of the electromagnetic current is similar to the symmetry of the quark mass matrix. The simplifications that come from $\delta m_{u}+\delta m_{d}+\delta m_{s}=0$ in the mass case are analogous to the simplifications we get from the identity $e_{u}+e_{d}+e_{s}=0$. A difference between quark mass and electromagnetic expansions is that in the mass expansion we can have both odd and even powers of $\delta m_{q}$, whereas only even powers of the quark charges $e_{q}$ are allowed. We consider contributions of $O\left(e_{q}^{2}\right)$ only. Hence, QED corrections can be simply read off from the mass expansion presented in [3], dropping the linear terms and changing masses to charges.

For the masses of octet mesons with the flavor structure $a \bar{b}$, and all annihilation diagrams

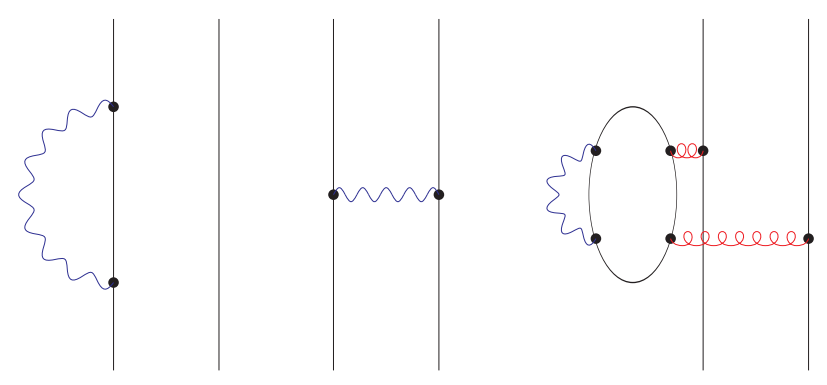

Figure 1: Examples of Feynman diagrams contributing to the meson electromagnetic mass to $O\left(e_{q}^{2}\right)$. Wavy lines are photons, curly lines are gluons. 
turned off, we find to $O\left(\delta m_{q}\right)$

$$
\begin{aligned}
M^{2}(a \bar{b}) & =M_{0}^{2}+\alpha\left(\delta m_{a}+\delta m_{b}\right)+\beta_{0}^{\mathrm{EM}}\left(e_{u}^{2}+e_{d}^{2}+e_{s}^{2}\right)+\beta_{1}^{\mathrm{EM}}\left(e_{a}^{2}+e_{b}^{2}\right)+\beta_{2}^{\mathrm{EM}}\left(e_{a}-e_{b}\right)^{2} \\
& +\gamma_{0}^{\mathrm{EM}}\left(e_{u}^{2} \delta m_{u}+e_{d}^{2} \delta m_{d}+e_{s}^{2} \delta m_{s}\right)+\gamma_{1}^{\mathrm{EM}}\left(e_{a}^{2} \delta m_{a}+e_{b}^{2} \delta m_{b}\right) \\
& +\gamma_{2}^{\mathrm{EM}}\left(e_{a}-e_{b}\right)^{2}\left(\delta m_{a}+\delta m_{b}\right)+\gamma_{3}^{\mathrm{EM}}\left(e_{a}^{2}-e_{b}^{2}\right)\left(\delta m_{a}-\delta m_{b}\right) \\
& +\gamma_{4}^{\mathrm{EM}}\left(e_{u}^{2}+e_{d}^{2}+e_{s}^{2}\right)\left(\delta m_{a}+\delta m_{b}\right)+\gamma_{5}^{\mathrm{EM}}\left(e_{a}+e_{b}\right)\left(e_{u} \delta m_{u}+e_{d} \delta m_{d}+e_{s} \delta m_{s}\right) .
\end{aligned}
$$

Several of the coefficients in (2) can be matched up with different classes of Feynman diagrams shown in Fig. 1. The first diagram, with both ends of the photon attached to the same valence quark, contributes to $\left(\beta_{1}^{\mathrm{EM}}+\beta_{2}^{\mathrm{EM}}\right)$. The second diagram, with the photon crossing between the valence lines, contributes to $\beta_{2}^{\mathrm{EM}}$. The last diagram, with the photon being attached to the sea quarks, is an example of a diagram contributing to $\beta_{0}^{\mathrm{EM}}$. It would be missed out if the electromagnetic field was quenched instead of dynamical. Similar assignments hold for the mixed (charge squared times mass) terms. For a single choice of sea quark masses, the $\beta_{0}^{\mathrm{EM}}$ and $\gamma_{4}^{\mathrm{EM}}$ terms can be absorbed into the constant $M_{0}^{2}$ and the $\alpha$ term. However, for a combined fit of both QCD and QCD + QED data we will need these coefficients. Similarly, for octet baryons with the flavor structure $a a b$ we find

$$
\begin{aligned}
M^{2}(a a b) & =M_{0}^{2}+\alpha_{1}\left(2 \delta m_{a}+\delta m_{b}\right)+\alpha_{2}\left(\delta m_{a}-\delta m_{b}\right) \\
& +\beta_{0}^{\mathrm{EM}}\left(e_{u}^{2}+e_{d}^{2}+e_{s}^{2}\right)+\beta_{1}^{\mathrm{EM}}\left(2 e_{a}^{2}+e_{b}^{2}\right)+\beta_{2}^{\mathrm{EM}}\left(e_{a}-e_{b}\right)^{2}+\beta_{3}^{\mathrm{EM}}\left(e_{a}^{2}-e_{b}^{2}\right) .
\end{aligned}
$$

This excludes the case of baryons with three different quarks, as in the $\Sigma^{0}-\Lambda$ system [5]. Again, the $\beta_{0}^{\mathrm{EM}}$ term can be absorbed into the mass term $M_{0}^{2}$.

Our goal is to compute the mass splittings of pseudoscalar mesons and octet baryons at the physical point for QCD + QED. This amounts to determining the coefficients $\alpha, \beta^{\mathrm{EM}}$ and $\gamma^{\mathrm{EM}}$ in (2) and (3). It greatly helps to vary valence and sea quark masses independently [3], which is referred to as partial quenching (PQ). In this case the sea quark masses remain constrained by $\bar{m}=$ constant, while the valence quark masses $\mu_{u}, \mu_{d}$ and $\mu_{s}$ are unconstrained. Defining $\delta \mu_{q}=\mu_{q}-\bar{m}$, the resulting mass formula for PQ octet mesons is

$$
\begin{aligned}
M^{2}(a \bar{b}) & =M_{0}^{2}+\alpha\left(\delta \mu_{a}+\delta \mu_{b}\right)+\beta_{0}^{\mathrm{EM}}\left(e_{u}^{2}+e_{d}^{2}+e_{s}^{2}\right)+\beta_{1}^{\mathrm{EM}}\left(e_{a}^{2}+e_{b}^{2}\right)+\beta_{2}^{\mathrm{EM}}\left(e_{a}-e_{b}\right)^{2} \\
& +\gamma_{0}^{\mathrm{EM}}\left(e_{u}^{2} \delta m_{u}+e_{d}^{2} \delta m_{d}+e_{s}^{2} \delta m_{s}\right)+\gamma_{1}^{\mathrm{EM}}\left(e_{a}^{2} \delta \mu_{a}+e_{b}^{2} \delta \mu_{b}\right) \\
& +\gamma_{2}^{\mathrm{EM}}\left(e_{a}-e_{b}\right)^{2}\left(\delta \mu_{a}+\delta \mu_{b}\right)+\gamma_{3}^{\mathrm{EM}}\left(e_{a}^{2}-e_{b}^{2}\right)\left(\delta \mu_{a}-\delta \mu_{b}\right) \\
& +\gamma_{4}^{\mathrm{EM}}\left(e_{u}^{2}+e_{d}^{2}+e_{s}^{2}\right)\left(\delta \mu_{a}+\delta \mu_{b}\right)+\gamma_{5}^{\mathrm{EM}}\left(e_{a}+e_{b}\right)\left(e_{u} \delta m_{u}+e_{d} \delta m_{d}+e_{s} \delta m_{s}\right) .
\end{aligned}
$$

For octet baryons the result is

$$
\begin{aligned}
M^{2}(a a b) & =M_{0}^{2}+\alpha_{1}\left(2 \delta \mu_{a}+\delta \mu_{b}\right)+\alpha_{2}\left(\delta \mu_{a}-\delta \mu_{b}\right) \\
& +\beta_{0}^{\mathrm{EM}}\left(e_{u}^{2}+e_{d}^{2}+e_{s}^{2}\right)+\beta_{1}^{\mathrm{EM}}\left(2 e_{a}^{2}+e_{b}^{2}\right)+\beta_{2}^{\mathrm{EM}}\left(e_{a}-e_{b}\right)^{2}+\beta_{3}^{\mathrm{EM}}\left(e_{a}^{2}-e_{b}^{2}\right) .
\end{aligned}
$$

The coefficients $\alpha, \beta^{\mathrm{EM}}$ and $\gamma^{\mathrm{EM}}$ in (4) and (5) are identical to those in (2) and (3). Hence, PQ calculations offer a computationally cheaper way of obtaining them.

In QCD + QED there is some ambiguity in the definition of the symmetric point. The definition we have chosen is that the electrically neutral pseudoscalar mesons have the same masses,

$$
M^{2}(u \bar{u})=M^{2}(d \bar{d})=M^{2}(s \bar{s})=M^{2}(d \bar{s})=M^{2}(s \bar{d})=M^{2}(n \bar{n}),
$$


where $n$ is a fictitious electrically neutral quark. As annihilation diagrams are neglected, different neutral mesons do not mix. We denote the Wilson hopping parameter $\kappa$ (defined in (10) below) marking the symmetric point by $\bar{\kappa}_{q}$. We then have

$$
\delta m_{q}=\left(m_{q}-\bar{m}\right)=1 / 2 \kappa_{q}^{\mathrm{sea}}-1 / 2 \bar{\kappa}_{q}, \quad \delta \mu_{q}=\left(\mu_{q}-\bar{m}\right)=1 / 2 \kappa_{q}^{\mathrm{val}}-1 / 2 \bar{\kappa}_{q} .
$$

It should be noted that even when all three quark masses are equal we do not have full SU(3) symmetry. Due to the different charges, the $u$ quark is always distinguishable from the $d$ and $s$ quark.

\section{Lattice matters}

The action we are using is

$$
S=S_{G}+S_{A}+S_{F}^{u}+S_{F}^{d}+S_{F}^{s} .
$$

Here $S_{G}$ is the tree-level Symanzik improved SU(3) gauge action, and $S_{A}$ is the noncompact U(1) gauge action [6, 7] of the photon,

$$
S_{A}=\frac{1}{2 e^{2}} \sum_{x, \mu<v}\left(A_{\mu}(x)+A_{v}(x+\mu)-A_{\mu}(x+v)-A_{\nu}(x)\right)^{2} .
$$

The fermion action for each flavor is

$$
\begin{array}{r}
\tilde{S}_{F}^{q}=\sum_{x}\left\{\frac{1}{2} \sum_{\mu}\left[\bar{q}(x)\left(\gamma_{\mu}-1\right) e^{-i e_{q} A_{\mu}(x)} \tilde{U}_{\mu}(x) q(x+\hat{\mu})-\bar{q}(x)\left(\gamma_{\mu}+1\right) e^{i e_{q} A_{\mu}(x)} \tilde{U}_{\mu}^{\dagger}(x-\hat{\mu}) q(x-\hat{\mu})\right]\right. \\
\left.+\frac{1}{2 \kappa_{q}} \bar{q}(x) q(x)-\frac{1}{4} c_{S W} \sum_{\mu \nu} \bar{q}(x) \sigma_{\mu \nu} F_{\mu \nu}(x) q(x)\right\},
\end{array}
$$

where $\tilde{U}_{\mu}$ is a single iterated mild stout smeared link [3]. The clover coefficient $c_{S W}$ has been computed nonperturbatively [8]. The quark charges are $e_{u}=2 / 3$ and $e_{d}=e_{s}=-1 / 3$. We presently neglect EM modifications to the clover term. This will leave us with corrections of $O\left(\alpha_{\mathrm{EM}} a\right)$, which are presumably smaller than the $O\left(a^{2}\right)$ corrections from QCD.

The action (8) is invariant under $\mathrm{U}(1)$ gauge transformations

$$
A_{\mu}(x) \rightarrow A_{\mu}(x)+\Delta_{\mu} \alpha(x), \quad q(x) \rightarrow e^{i e_{q} \alpha(x)} q(x) .
$$

This is not the case for propagators of charged particles, which demands fixing the gauge, as in perturbation theory. We choose the Landau gauge, which is defined by the condition

$$
\bar{\Delta}_{\mu} A_{\mu}(x)=0 \text {. }
$$

$\Delta_{\mu}\left(\bar{\Delta}_{\mu}\right)$ is the forward (backward) lattice derivative. The Landau gauge does not eliminate all gauge degrees of freedom, but allows for shifts $\Delta_{\mu} \alpha(x)$ of the photon field with [7]

$$
\Delta^{2} \alpha(x)=0,
$$


where $\Delta^{2}=\Delta_{\mu} \bar{\Delta}_{\mu}$. To maintain (anti-)periodicity of the quark fields, $\alpha(x)$ must be of the form

$$
e_{q} \alpha(x)=\sum_{\mu} \frac{2 \pi}{L_{\mu}} n_{\mu} x_{\mu}, \quad n_{\mu} \in \mathbb{Z},
$$

where $L_{\mu}$ is the extent of the lattice in $\mu$ direction. This gauge field redundancy can be eliminated by adding multiples of $2 \pi / e_{q} L_{\mu}$ to $A_{\mu}(x)$, such that

$$
-\frac{\pi}{\left|e_{q}\right| L_{\mu}}<B_{\mu} \leq \frac{\pi}{\left|e_{q}\right| L_{\mu}}, \quad B_{\mu}=\frac{1}{V} \sum_{x} A_{\mu}(x) .
$$

Taking $e_{q}=-1 / 3$ in (14) serves both charges. This procedure leaves Polyakov loops and fermion determinants unchanged for all quarks.

In a constant background field $B_{\mu}$ the correlator of a single hadron $H$ becomes [7]

$$
\langle 0|H(t) \bar{H}(0)| 0\rangle \simeq\left|Z_{H}\right|^{2} e^{-\sqrt{M_{H}^{2}+\left(\vec{p}+e_{H} \vec{B}\right)^{2}} t} e^{-i e_{H} B_{4} t},
$$

where $M_{H}, \vec{p}$ and $e_{H}$ are mass, three-momentum and electric charge of the hadron. This amounts to a shift of the rest mass of the charged hadrons, $M_{H}^{2} \rightarrow M_{H}^{2}+e_{H}^{2} \vec{B}^{2}$. The BMW collaboration [9] puts $\sum_{\vec{x}} A_{\mu}\left(\vec{x}, x_{4}\right)=0$ for all $x_{4}, \mu$ [10], which would mean that valence and sea quarks feel different $\mathrm{U}(1)$ fields.

The strategy is to simulate at an artificial coupling $e^{2}=1.25$, and then interpolate between this point and pure QCD to the physical fine structure constant $\alpha_{\mathrm{EM}}=1 / 137$. This value is chosen so that electromagnetic effects can be easily seen, but is still small enough that $O\left(e^{4}\right)$ effects are negligible. The strong coupling constant is chosen to be $\beta=5.50$, where we have our largest sample of dynamical QCD configurations [11].

In this Letter we restrict ourselves to simulations at the symmetric point, $\delta m_{u}=\delta m_{d}=\delta m_{s}=$ 0 , which we define as $X_{\pi}^{2} / X_{N}^{2}=0.126$, where $X_{N}^{2}=\left(M_{n}^{2}+M_{p}^{2}+M_{\Sigma^{-}}^{2}+M_{\Sigma^{+}}^{2}+M_{\Xi^{-}}^{2}+M_{\Xi^{0}}^{2}\right) / 6$. We may use either $X_{\pi}$ or $X_{N}$ to set the scale. After several tuning runs carried out on $24^{3} \times 48$ lattices we arrived at the $\kappa$ values

$$
\bar{\kappa}_{u}=0.124362, \quad \bar{\kappa}_{d}=\bar{\kappa}_{s}=0.121713 .
$$

Our present ensembles are listed in Table 1, with $O(2000)$ to $O(500)$ trajectories. On these ensembles we have computed PQ pseudoscalar meson and octet baryon masses for a variety of quark masses ranging from $m_{P S} / m_{N}=0.22$ to 0.5 , with $e_{q}=-1 / 3,0$ and $+2 / 3$. This gave us about 40 pseudoscalar masses and 70 baryon masses per ensemble. The baryons include several artificial states containing the fictitious $n$ quark and charge 2 baryons with flavor structure $u и u^{\prime}$.

\begin{tabular}{c|c|c|c|c|c|l}
$\beta$ & $e^{2}$ & $V$ & $\kappa_{u}$ & $\kappa_{d}$ & $\kappa_{s}$ & \multicolumn{1}{|c}{$\vec{B}^{2}$} \\
\hline 5.50 & 1.25 & $24^{3} \times 48$ & 0.124362 & 0.121713 & 0.121713 & 0.024 \\
5.50 & 1.25 & $32^{3} \times 64$ & 0.124362 & 0.121713 & 0.121713 & 0.0079 \\
5.50 & 1.25 & $48^{3} \times 96$ & 0.124362 & 0.121713 & 0.121713 & 0.000095
\end{tabular}

Table 1: Parameters of our data ensembles, together with the background field. 


\section{Results}

In contrast to QCD, equal meson masses at the symmetric point no longer mean equal bare quark masses. We renormalize the quark masses to remove this defect. We do so by absorbing the QED terms of the neutral pseudoscalar mesons into the quark self-energies. On our symmetric background, $\delta m_{u}=\delta m_{d}=\delta m_{s}=0$, this is achieved by replacing $\delta \mu_{q}$ by the 'Dashen' scheme mass [12]

$$
\delta \mu_{q}^{D}=\left[1+\left(\gamma_{1}^{\mathrm{EM}} / \alpha\right) e_{q}^{2}\right] \delta \mu_{q} .
$$

Substituting (17) into (4), and absorbing $\beta_{0}^{\mathrm{EM}}$ into $M_{0}^{2}$ and $\gamma_{4}^{\mathrm{EM}}$ into $\alpha$, we obtain in the 'Dashen' scheme

$$
\begin{aligned}
M^{2}(a \bar{b}) & =M_{0}^{2}+\alpha\left(\delta \mu_{a}^{D}+\delta \mu_{b}^{D}\right)+\beta_{2}^{\mathrm{EM}}\left(e_{a}-e_{b}\right)^{2} \\
& +\gamma_{2}^{\mathrm{EM}}\left(e_{a}-e_{b}\right)^{2}\left(\delta \mu_{a}^{D}+\delta \mu_{b}^{D}\right)+\gamma_{3}^{\mathrm{EM}}\left(e_{a}^{2}-e_{b}^{2}\right)\left(\delta \mu_{a}^{D}-\delta \mu_{b}^{D}\right) .
\end{aligned}
$$

Note that $\beta_{1}^{\mathrm{EM}}=0$ by definition, eq. (6). We define the crtical point to be the point where the masses of the neutral pseudoscalar mesons vanish. It is then easily seen that the 'Dashen' scheme quark masses are all equal at the symmetric point, $\bar{\mu}_{q}^{D}=M_{0}^{2} / 2 \alpha, q=u, d, s$ and $n$. It follows that the electromagnetic contributions to the neutral pseudoscalar meson masses, $M_{\pi^{0}}$ and $M_{K^{0}}$, are zero. To be consistent, we also expand the baryon masses in terms of the 'Dashen' masses,

$$
\begin{aligned}
M^{2}(a a b) & =M_{0}^{2}+\alpha_{1}\left(2 \delta \mu_{a}^{D}+\delta \mu_{b}^{D}\right)+\alpha_{2}\left(\delta \mu_{a}^{D}-\delta \mu_{b}^{D}\right) \\
& +\beta_{1}^{\mathrm{EM}}\left(2 e_{a}^{2}+e_{b}^{2}\right)+\beta_{2}^{\mathrm{EM}}\left(e_{a}-e_{b}\right)^{2}+\beta_{3}^{\mathrm{EM}}\left(e_{a}^{2}-e_{b}^{2}\right) .
\end{aligned}
$$

The background field $B$ may give rise to significant finite size effects, which need to be corrected for before fitting the data. In Fig. 2 we compare the effect with finite volume QED corrections from effective field theory [13] on the $32^{3} \times 64$ lattice. Both effects turn out to be of the same order of magnitude, but of opposite sign. Similar results hold for the $24^{3} \times 48$ lattice, while the effect is practically negligible on the $48^{3} \times 96$ lattice. We correct for this effect by subtracting $e_{H}^{2} \vec{B}^{2}$ from each lattice mass before fitting.

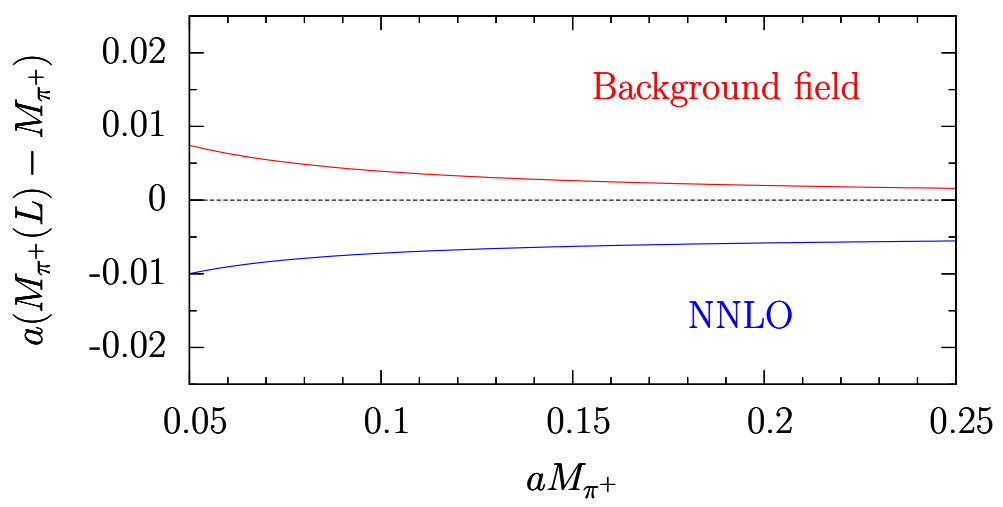

Figure 2: Finite volume corrections to the pion mass as a function of $M_{\pi}$ on the $32^{3} \times 64$ lattice caused by the background field (Table 1), compared to the NNLO prediction of Davoudi and Savage [13]. 

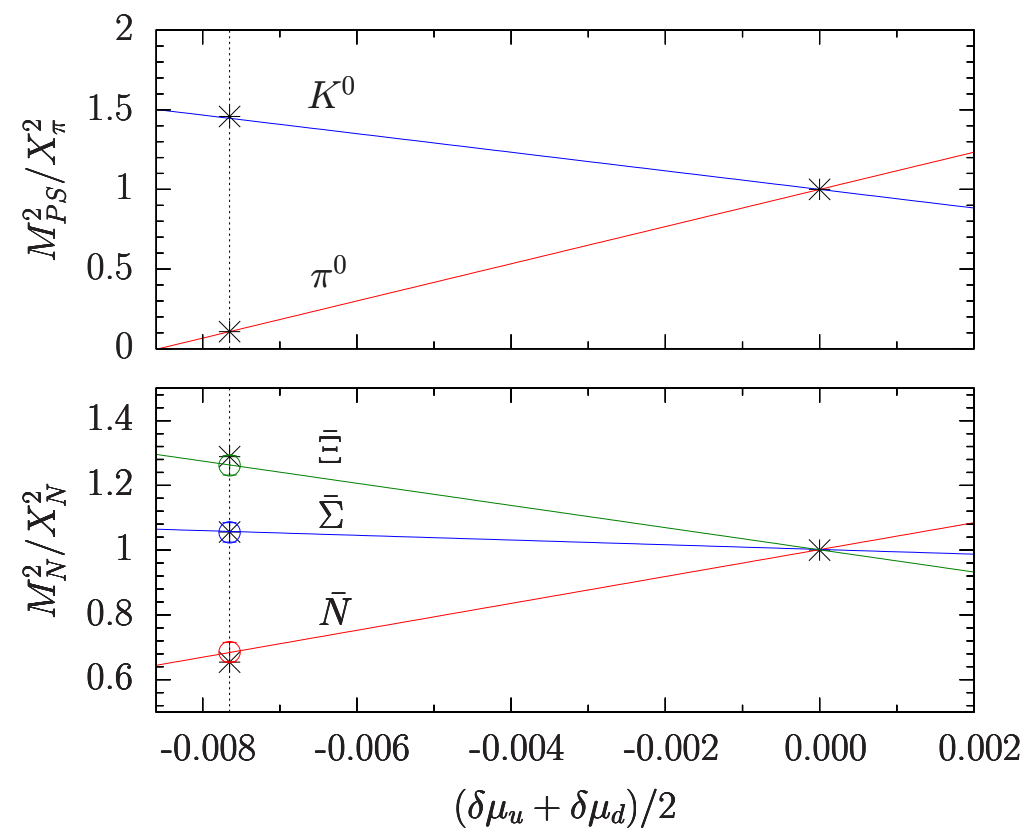

Figure 3: Fan plots of pseudoscalar meson (top) and baryon masses (bottom) on the $48^{3} \times 96$ lattice as a function of $\delta \mu_{u}+\delta \mu_{d}$. The baryon masses are the averages of the isospin doublets.

It turns out that the $\kappa$ values (16) do not quite satisfy the constraint (6). A more accurate estimate can be determined from a fit to the pseudoscalar meson masses. On the $48^{3} \times 96$ lattice we obtain

$$
\bar{\kappa}_{u}=0.124382, \quad \bar{\kappa}_{d}=\bar{\kappa}_{s}=0.121703, \quad \bar{\kappa}_{n}=0.120814,
$$

which is only a small displacement. We shall expand about these $\kappa$ values in our subsequent fits. For the total contribution of QCD + QED it does not matter which scheme we use to define the quark masses, but for the individual contributions of QCD and QED it will make a difference. The fits of (18) and (19) to the lattice data are quite robust, giving $\chi^{2} /$ dof $=0.7-1.2$. To obtain physical numbers we extrapolate the coefficients $\beta_{i}^{\mathrm{EM}}$ and $\gamma_{i}^{\mathrm{EM}}$ to $\alpha_{\mathrm{EM}}=1 / 137$ by scaling them with a factor $10 / 137$. In our extrapolation to the physical point we keep the sum of the quark masses constant. We choose $M_{\pi^{0}}^{2}$ and $M_{K^{0}}^{2}-M_{K^{+}}^{2}+M_{\pi^{+}}^{2}-M_{\pi^{0}}^{2}$ to determine the physical $\kappa$ values. In Fig. 3 we show the result of the fit to the meson and baryon masses on the $48^{3} \times 96$ lattice. We obtain $X_{\pi}^{2} / X_{N}^{2}=0.128(3)$, which is to be compared with the physical value, 0.126 . This tells us that we have hit the symmetric point with remarkable precision. The lattice spacing turns out to be $a=0.068(2) \mathrm{fm}$. The baryon masses extrapolate nicely to their experimental values. Similarly good results are found on the $32^{3} \times 64$ lattice. Having found the $\kappa$ values of the physical point and the point where the 'Dashen' scheme masses vanish (the critical point), we can determine the quark masses. For the quark mass ratios we find on the $48^{3} \times 96$ lattice in the 'Dashen' scheme

$$
\frac{m_{u}}{m_{d}}=0.52(2), \quad \frac{m_{s}}{m_{d}}=19.7(9) .
$$

In this Letter we are primarily interested in the isospin splittings of pseudoscalar meson and octet baryon masses. As it is well known that QED will induce additional finite size effects, we present in Fig. 4 our results for the isospin splittings as a function of lattice size. The difference 

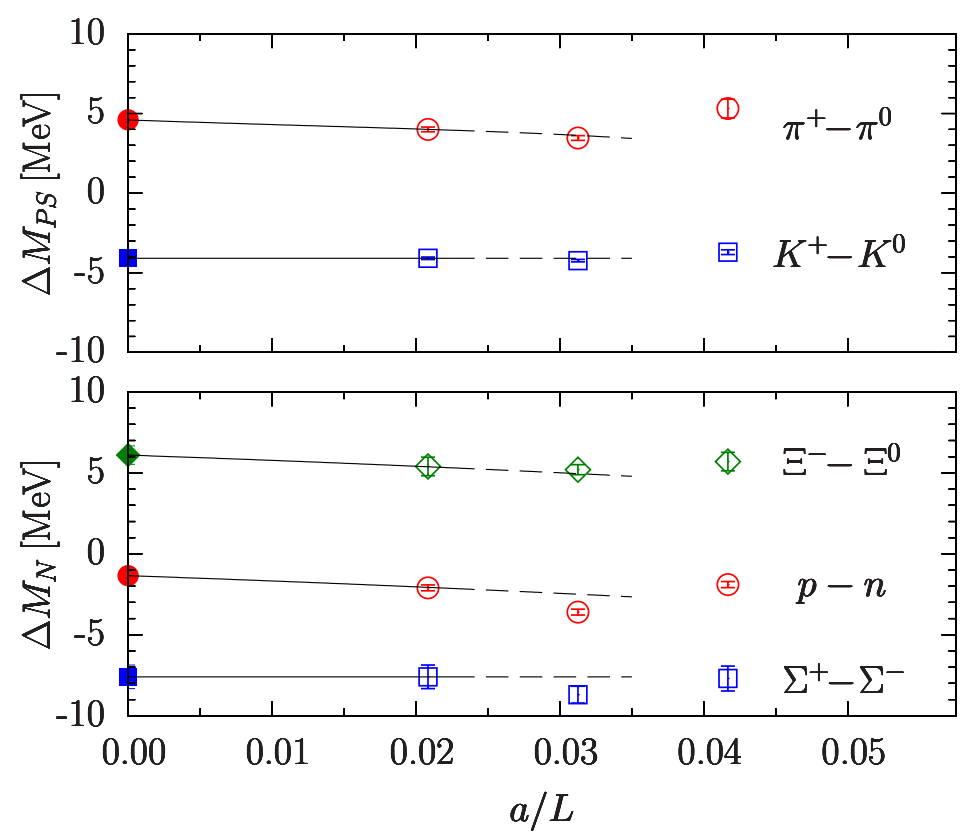

Figure 4: Mass splittings of pseudoscalar meson (top) and baryon masses (bottom) as a function of the spatial size of the lattice. The numbers on the largest volume have been extrapolated to infinite volume using [13].

$M_{K^{0}}-M_{K^{+}}$is not expected to depend much on the volume, as it was used to fix the $\kappa$ values. The curves represent the finite size corrections predicted by the chiral effective theory [13]. They have been drawn through the points on the $48^{3} \times 96$ lattice. Assuming that the predictions are valid up to this volume, we use these expressions to extrapolate our results to the infinite volume. The results on the smaller volumes show some convergence towards these curves. In Table 2 we give our results for the mass splittings in the infinite volume, for the total and the QED contribution separately. The QED contribution refers to the 'Dashen' scheme. The traditional way of expressing the electromagnetic contributions is through $\Delta_{\pi}=M_{\pi^{+}}^{2}-M_{\pi^{0}}^{2}$ and the $\epsilon$ parameter,

$$
\left(M_{K^{+}}^{2}-M_{K^{0}}^{2}\right)_{\mathrm{QED}}-M_{\pi^{+}}^{2}+M_{\pi^{0}}^{2}=\epsilon \Delta_{\pi} .
$$

On the $48^{3} \times 96$ lattice we find

$$
\epsilon=0.49(3)
$$

In Table 2 we also quote the experimental mass splittings, where we observe good agreement for the pion, kaon and nucleon. The mass splittings of $\Sigma$ and $\Xi$ turn out to be somewhat lower than experiment (in absolute terms), but still agree within the errors. Both, the total and the electromagnetic contributions satisfy the Coleman-Glashow relation [14]. So do the experimental numbers, which a posteriori supports our ansatz (19). The QED contribution to the $n-p$ mass splitting turns out to be significantly larger (in absolute terms) than expected from the Cottingham formula [15].

As discussed in the introduction, the existence of the Universe as we know it is highly sensitive to the size of the $n-p$ mass difference. Having an analytic expression for the nucleon mass as a function of the quark masses and $\alpha_{\mathrm{EM}}$, we can visualize the allowed region, as shown in Fig. 5. It appears that both $\alpha_{\mathrm{EM}}$ and the light quark masses are finely tuned to each other. On 


\begin{tabular}{c|c|c|c}
$\Delta M$ & QCD + QED & QED & Experiment \\
\hline$M_{\pi^{+}}-M_{\pi^{0}}$ & $4.60(20)$ & $4.60(20)$ & 4.59 \\
$M_{K^{0}}-M_{K^{+}}$ & $4.09(10)$ & $-1.66(6)$ & 3.93 \\
$M_{n}-M_{p}$ & $1.35(18)$ & $-2.29(21)$ & 1.30 \\
$M_{\Sigma^{-}}-M_{\Sigma^{+}}$ & $7.60(73)$ & $-0.63(6)$ & 8.08 \\
$M_{\Xi^{-}}-M_{\Xi^{0}}$ & $6.10(57)$ & $1.26(12)$ & 6.85
\end{tabular}

Table 2: Mass splittings in the infinite volume, in units of $\mathrm{MeV}$.

the horizontal axis $\left(M_{n}-M_{p}\right)_{Q C D} \propto\left(m_{d}-m_{u}\right)$. This excludes $m_{u}=m_{d}$ and puts a lower bound on $m_{u}$. When expressed in terms of the mass ratio $m_{u} / m_{d}$, the allowed region translates into a narrow band around $m_{u} / m_{d}=0.5$. This fits in with previous results from QCD. The region between the two vertical lines in Fig. [5 is the prediction of [16] for $\left(M_{n}-M_{p}\right)_{Q C D}$. The kaon mass difference that enters the calculation has been taken from Table 2. Comparing this result with our present calculation, indicated by the solid circle, we find good agreement between the two results, which supports a rather larger electromagnetic contribution to $M_{n}-M_{p}$ than predicted by effective theory.

\section{Conclusion and outlook}

We have outlined a program to systematically investigate the flavor structure of hadrons in QCD + QED, and successfully computed the isospin splittings of pseudoscalar meson and octet baryon masses. To corroborate our results, we plan to do simulations along the $\bar{m}=$ const line towards

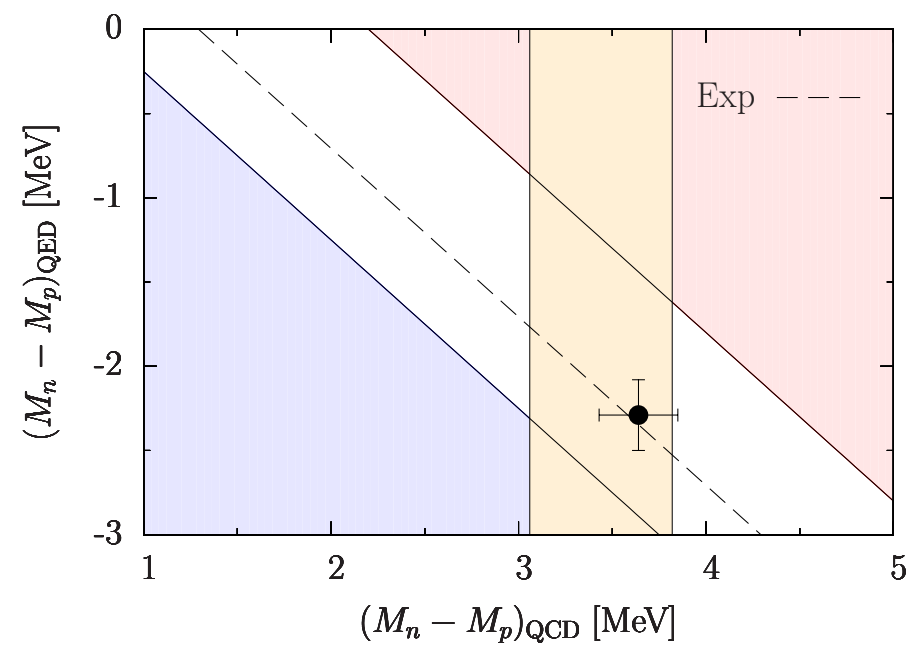

Figure 5: The mass difference of neutron and proton divided into pure QCD and QED contributions. The dashed line is the experimental value, the solid circle is our result. The shaded region of no $\beta$ decay is to the bottom left, the no fusion region to the top right. The shaded region between the vertical lines is the prediction of pure QCD. 
the physical point and at smaller lattice spacing in the near future.

Our results on three different volumes indicate that finite size effects cannot be accounted for correctly by the chiral effective theory [13] for $L \lesssim 2.5 \mathrm{fm}$. This is also not to be expected, as the zero modes are not removed completely in our case, nor can they be removed in a gauge invariant manner, but keep residing in the background field. An appropriate description of finite volume corrections in periodic QED is still missing.

\section{Acknowledgment}

This work has been partly supported by DFG through grant Schi 422/10-1 and the the Australian Research Council through grants DP140103067 (RDY and JMZ), FT120100821 (RDY) and FT100100005 (JMZ). The numerical calculations were carried out on the BlueGeneQs at FZ Jülich and EPCC Edinburgh (using DIRAC 2), the Cray XC30 at HLRN (Berlin and Hannover) and on the NCI National Facility (Canberra).

\section{References}

[1] For recent work see: T. Blum, R. Zhou, T. Doi, M. Hayakawa, T. Izubuchi, S. Uno and N. Yamada, Phys. Rev. D 82 (2010) 094508 [arXiv:1006.1311 [hep-lat]], S. Aoki, K.-I. Ishikawa, N. Ishizuka, K. Kanaya, Y. Kuramashi, Y. Nakamura, Y. Namekawa, M. Okawa, Y. Taniguchi, A. Ukawa, N. Ukita and T. Yoshie, Phys. Rev. D 86 (2012) 034507 [arXiv:1205.2961 [hep-lat]], Sz. Borsanyi, S. Dürr, Z. Fodor, J. Frison, C. Hoelbling, S. D. Katz, S. Krieg, Th. Kurth, L. Lellouch, Th. Lippert, A. Portelli, A. Ramos, A. Sastre and K. Szabo, Phys. Rev. Lett. 111 (2013) 25, 252001 [arXiv:1306.2287 [hep-lat]], G. M. de Divitiis, R. Frezzotti, V. Lubicz, G. Martinelli, R. Petronzio, G. C. Rossi, F. Sanfilippo, S. Simula and N. Tantalo, Phys. Rev. D 87 (2013) 11, 114505 [arXiv:1303.4896 [hep-lat]], R. Horsley, Y. Nakamura, D. Pleiter, P. E. L. Rakow, G. Schierholz, H. Stüben, R. D. Young and J. M. Zanotti, PoS Lattice 2013 (2014) 499 [arXiv:1311.4554 [hep-lat]], R. Zhou and S. Gottlieb, PoS LATTICE 2014 (2014) 024 [arXiv:1411.4115 [hep-lat], Sz. Borsanyi, S. Dürr, Z. Fodor, C. Hoelbling, S. D. Katz, S. Krieg, L. Lellouch, T. Lippert, A. Portelli, K. K. Szabo and B. C. Toth, Science 347 (2015) 1452 [arXiv:1406.4088 [hep-lat]].

[2] W. Bietenholz, V. Bornyakov, N. Cundy, M. Göckeler, R. Horsley, A. D. Kennedy, W. G. Lockhart and Y. Nakamura, H. Perlt, D. Pleiter, P. E. L. Rakow, A. Schäfer, G. Schierholz, A. Schiller, H. Stüben and J. M. Zanotti, Phys. Lett. B 690 (2010) 436 [arXiv:1003.1114 [hep-lat]].

[3] W. Bietenholz, V. Bornyakov, M. Göckeler, R. Horsley, W. G. Lockhart, Y. Nakamura, H. Perlt and D. Pleiter, P. E. L. Rakow, G. Schierholz, A. Schiller, T. Streuer, H. Stüben, F. Winter and J. M. Zanotti, Phys. Rev. D 84 (2011) 054509 [arXiv:1102.5300 [hep-lat]].

[4] R. Horsley, Y. Nakamura, D. Pleiter, P. E. L. Rakow, G. Schierholz, H. Stüben, R. D. Young and J. M. Zanotti, PoS Lattice 2013 (2014) 499 [arXiv:1311.4554 [hep-lat]]. 
[5] R. Horsley, J. Najjar, Y. Nakamura, H. Perlt, D. Pleiter, P. E. L. Rakow, G. Schierholz, A. Schiller, H. Stüben and J. M. Zanotti, Phys. Rev. D 91 (2015) 7, 074512 [arXiv:1411.7665 [hep-lat]].

[6] M. Göckeler, R. Horsley, E. Laermann, P. E. L. Rakow, G. Schierholz, R. Sommer and U. J. Wiese, Nucl. Phys. B 334 (1990) 527.

[7] M. Göckeler, R. Horsley, P. E. L. Rakow, G. Schierholz and R. Sommer, Nucl. Phys. B 371 (1992) 713.

[8] N. Cundy, M. Göckeler, R. Horsley, T. Kaltenbrunner, A. D. Kennedy, Y. Nakamura, H. Perlt, D. Pleiter, P. E. L. Rakow, A. Schäfer, G. Schierholz, A. Schiller, H. Stüben and J. M. Zanotti, Phys. Rev. D 79 (2009) 094507 [arXiv:0901.3302 [hep-lat]].

[9] S. Borsanyi, S. Dürr, Z. Fodor, C. Hoelbling, S. D. Katz, S. Krieg, L. Lellouch, T. Lippert, A. Portelli, K. K. Szaboand B. C. Toth, Science 347 (2015) 1452 [arXiv:1406.4088 [heplat]].

[10] M. Hayakawa and S. Uno, Prog. Theor. Phys. 120 (2008) 413 [arXiv:0804.2044 [hep-ph]].

[11] V. G. Bornyakov, R. Horsley, R. Hudspith, Y. Nakamura, H. Perlt, D. Pleiter, P. E. L. Rakow, G. Schierholz, A. Schiller, H. Stüben and J. M. Zanotti, arXiv:1508.05916 [hep-lat].

[12] R. Horsley, Y. Nakamura, H. Perlt and D. Pleiter, P. E. L. Rakow, G. Schierholz, A. Schiller, H. Stüben, R. D. Young and J. M. Zanotti, QED effects in the pseudoscalar meson sector, to be published.

[13] Z. Davoudi and M. J. Savage, Phys. Rev. D 90 (2014) 054503 [arXiv:1402.6741 [hep-lat]].

[14] S. R. Coleman and S. L. Glashow, Phys. Rev. Lett. 6 (1961) 423.

[15] J. Gasser and H. Leutwyler, Nucl. Phys. B 94 (1975) 269, A. Walker-Loud, C. E. Carlson and G. A. Miller, Phys. Rev. Lett. 108 (2012) 232301 [arXiv:1203.0254 [nucl-th]], F. B. Erben, P. E. Shanahan, A. W. Thomas and R. D. Young, Phys. Rev. C 90 (2014) 6, 065205 [arXiv:1408.6628 [nucl-th]], J. Gasser, M. Hoferichter, H. Leutwyler and A. Rusetsky, Eur. Phys. J. C 75 (2015) 8, 375 [arXiv:1506.06747 [hep-ph]].

[16] R. Horsley, J. Najjar, Y. Nakamura, D. Pleiter, P. E. L. Rakow, G. Schierholz and J. M. Zanotti, Phys. Rev. D 86 (2012) 114511 [arXiv:1206.3156 [hep-lat]]. 